\title{
Predicting the Spectral Effects of Soils on High Concentrating Photovoltaic Systems
}

\author{
Patrick D. Burton ${ }^{\mathrm{a}, *}$, Bruce H. King ${ }^{\mathrm{b}}$, Daniel Riley ${ }^{\mathrm{b}}$ \\ ${ }^{a}$ Chemical and Biological Systems Department, Sandia National Laboratories, Albuquerque NM 87185-0734 USA \\ ${ }^{b}$ Photovoltaic and Distributed Systems Integration Department, Sandia National Laboratories, Albuquerque NM 87185-0951 USA
}

\section{Abstract}

Soiling losses on high concentrating photovoltaic (HCPV) systems may be influenced by the spectral properties of accumulated soil. We have predicted the response of an isotype cell to changes in spectral content and reduction in transmission due to soiling using measured UV/vis transmittance through soil films. Artificial soil test blends deposited on glass coupons were used to supply the transmission data, which was then used to calculate the effect on model spectra. The wavelength transparency of the test soil was varied by incorporating red and yellow mineral pigments into graded sand. The more spectrally responsive (yellow) soils were predicted to alter the current balance between the top and middle subcells throughout a range of air masses corresponding to daily and seasonal variation.

Keywords: Spectral response, triple junction limiting current, soiling losses

\section{Introduction}

High-concentrating photovoltaic (HCPV) systems are carefully engineered systems designed to capture as much of the direct (non-scattered) portion of the solar spectrum as possible. Precise sun-tracking hardware and algorithms enable highconcentration optics to focus this light onto triple junction (TJ) receivers, all to improve the efficiency, energy density and cost effectiveness of the system. As a result of this complexity, small deviations from optimal conditions propagate through the system and reduce the overall performance. Due to the substantial capital costs of HCPV systems, any loss in performance is a significant issue. Even small losses can propagate to significant costs, as discussed by Mingguo et al. (2013). In addition to well-known loss mechanisms such as tracking error (Muller, 2009; Stafford et al., 2009) and cloud cover (Viana et al., 2011), the triple junction (TJ) cells used in HCPV systems are sensitive to the uniformity and spectral content of the available light (Victoria et al., 2013). Baig et al. (2012) presented a discussion of non-uniform illumination due to tracking error, collector optical imperfections and spectral response. Of these, spectral response represents a particular challenge. All concentrating optics introduce some spectral effect as they transmit light (Victoria et al., 2013; Cotal and Sherif, 2005); however, environmental factors can exacerbate the effect. Gueymard (2009) has discussed the sensitivity of HCPV systems to spectral variations due to airmass in terms of both elevation of the site and atmospheric aerosol content. In addition to aerosol particles, accumulated soil on the receiver can both absorb and scatter the incident light. Soil decreases photocurrent by reducing the transmission of some wavelengths of light through the optical

\footnotetext{
${ }^{*}$ Corresponding author: +1 (505)284-1543

Email addresses: pdburto@sandia.gov (Patrick D. Burton), bhking@sandia.gov (Bruce H. King), driley@sandia.gov (Daniel Riley)
}

elements of the device. The total electrical output of HCPV systems has been shown (Vivar et al., 2010) to be more sensitive to soiling than flat plate PV due to the reduction in light reaching the device. However; some wavelengths of light can scatter through the soil, resulting in an altered spectrum at the receiver. The spectral content of the available light influences the limiting current of series-connected TJ devices on a daily and seasonal basis (Torrey et al., 2011).

Soiling effects in the literature have typically emphasized loss in transmission due to the total mass of accumulated material (Miller and Kurtz, 2011; El-Shobokshy et al., 1985). Specific properties of the soil, and thereby spectral quality of transmitted light, have not been discussed in detail with regards to HCPV systems. However, we have shown that the spectrum of transmitted light through soiled glass is dependent upon soil composition (Burton and King, 2014b). A neutral density soil would cause predictable losses in the total system output. A wavelength-sensitive soil could induce current mismatch between subcells in TJ cells.

Since many reports discuss the importance of spectral content to HCPV systems, a theoretical study was undertaken to predict the effect of observed light transmission through soil on TJ cells. Emphasis was placed on understanding the effect of soil as an optical element of any HCPV system, so the soil was treated as a stand-alone element. In addition to the magnitude of the soil effect at standard (AM1.5D) conditions, changes in the incident light throughout a typical day and year were considered. Performance predictions of soiled HCPV could improve the expected cost effectiveness of cleaning regimens, or spectrum enhancing technologies such as secondary optics. For example, Victoria et al. (2013) have shown improved spectral tolerance by using secondary optics, but noted the additional manufacturing cost is a significant consideration. A more thorough understanding of the performance changes induced by soil

November 14, 2014 
could provide insight to the cost effectiveness of soil mitigation technologies, or O\&M costs for large HCPV plants. In the present work, we examine the potential spectral effects of soil analogues typical of the US southwest on calibrated isotype cell data. The change in spectral response was used to predict the limiting current condition of an HCPV device. Premature current limiting behavior, relative to performance at standard conditions, was considered as a system loss. Performance predictions of HCPV devices were made using measurements from prior work (Burton and King, 2014b).

\section{Methods}

This work used experimentally collected transmission data (Burton and King, 2014b) to predict the spectral response of TJ $\mathrm{GaInP} / \mathrm{GaInAs} / \mathrm{Ge}$ (noted as Top/Middle/Bottom junctions, respectively) photovoltaic devices. Light transmission through a soil film previously collected by UV/vis spectroscopy (Burton and King, 2014b) was used to simulate direct normal irradiance (DNI) reaching the HCPV cell. These measured values were used to calculate the expected short circuit current density $\left(J_{\mathrm{SC}}\right)$ of a hypothetical device under a similar soil coverage. A nominal baseline for DNI spectral behavior was determined by multiplying the standard AM1.5D spectrum (ASTM, 2012) by the reported device response. The percent transmission due to each soil type and mass loading measured by the spectrophotometer was applied as a reduction factor to the calculated baseline, as shown in Eq. (1).

The subcell spectral response $(S R(\lambda))$ of an isotype spectral sensor (BPI-IT1, Black Photon International) at each calibrated wavelength was used to predict the effects of soil on a TJ device. In the isotype sensor, each independent subcell collects the full spectrum allowed by its respective bandgap; however, only the active cell is connected to an external circuit. As a result, the current reported by each subcell is exaggerated compared to the performance of a series-connected device, which would be limited to the current of the lowest output subcell. Since the emphasis of this study was the relative impact of various soil types on TJ devices, an artificial constraint was imposed by scaling the middle cell response to equal the top cell at AM1.5D. A multiplicative factor of $90.68 \%$ was applied to the integrated area of the middle subcell.

The baseline was calculated as the integral over wavelength $(\lambda)$ of the standard AM1.5D spectrum $\left(E_{A S T M}(\lambda)\right)$ multiplied by the $S R(\lambda)$ of each subcell. The ratio of $J_{\mathrm{SC}}^{\mathbf{T}, A S T M} / J_{\mathrm{SC}}^{\mathbf{M}, A S T M}$ was applied to subsequent calculations for the middle subcell under soiled conditions, as shown in Eq. (2).

$$
\begin{array}{r}
J_{\mathrm{SC}}^{T, \text { soiled }}=\int_{\lambda_{T_{1}}}^{\lambda_{T_{2}}} E_{x}(\lambda) \cdot S R(\lambda) \cdot \% T(\lambda) d \lambda \\
J_{\mathrm{SC}}^{M, \text { soiled }}=\frac{J_{\mathrm{SC}}^{T, A S T M}}{J_{\mathrm{SC}}^{M, A S T M}} \int_{\lambda_{M_{1}}}^{\lambda_{M_{2}}} E_{x}(\lambda) \cdot S R(\lambda) \cdot \% T(\lambda) d \lambda
\end{array}
$$

The effect of soil was calculated by multiplying the spectrum by the measured light transmission $(\% T(\lambda))$ to account for the light reaching the device. Changes in spectrum throughout a typical day were predicted using a DNI spectrum $\left(E_{A M}(\lambda)\right)$ calculated using the Simple Model of the Atmospheric Radiative Transfer of Sunshine (SMARTS) (Gueymard, 1995, 2001). The SMARTS inputs for an ASTM 173-G spectrum were used over a range between AM1.0D to AM15.0D in 0.5 increments to roughly approximate the spectrum of a typical day. Each spectrum was used as an input as described in Eq. (2). A similar approach has been described by Qasem et al. (2012) to evaluate the effects of tilt angle on flat-plate systems. Seasonal effects were modeled using the extreme conditions of each season; i.e. the solstices, to capture the greatest possible variation between data sets. In order to calculate the spectra for specific dates, the standard inputs were replaced with location-specific data collected in Albuquerque, NM $\left(35.05^{\circ} \mathrm{N}, 106.54^{\circ} \mathrm{W}\right)$. Summer and winter solstice calculations were performed using SMARTS with inputs modified for atmospheric data measured on 12/21/2012 and 06/20/2013. The standard SMARTS inputs were adjusted to account for local azimuth, zenith, air temperature, relative humidity and air pressure. Other variables, such as aerosol optical depth, were held constant at reference conditions, similar to the technique reported by King et al. (2011). Spectra $\left(E_{\text {solstice }}(\lambda)\right)$ were calculated at one minute intervals over the range of available data for each day.

\section{Results and Discussion}

DNI transmission through soiled surfaces was modeled using measurements from UV/vis spectroscopy. Soil blends consist of $60 \mathrm{wt} . \%$ graded sand, $40 \mathrm{wt} . \%$ mineral pigment and trace amounts of soot. The mineral pigments are noted as a ratio of weight percent $\mathrm{Fe}_{2} \mathrm{O}_{3}$ to göthite; 40:0 indicating 40 wt.\% $\mathrm{Fe}_{2} \mathrm{O}_{3}$, and 30:10 indicating a mixture of $30 \mathrm{wt} . \% \mathrm{Fe}_{2} \mathrm{O}_{3}$ and 10 wt.\% göthite, etc. Representative grime samples are shown in FIG. 1 to contrast the göthite-rich (10:30 and 0:40 blends) with $\mathrm{Fe}_{2} \mathrm{O}_{3}$-rich blends (30:10 and 40:0). Grime containing red $\mathrm{Fe}_{2} \mathrm{O}_{3}$ functioned as a nearly neutral density filter in the wavelength region relevant to most flat plate PV systems (300$1800 \mathrm{~nm}$ ). The transmission decreases over the near infrared re-

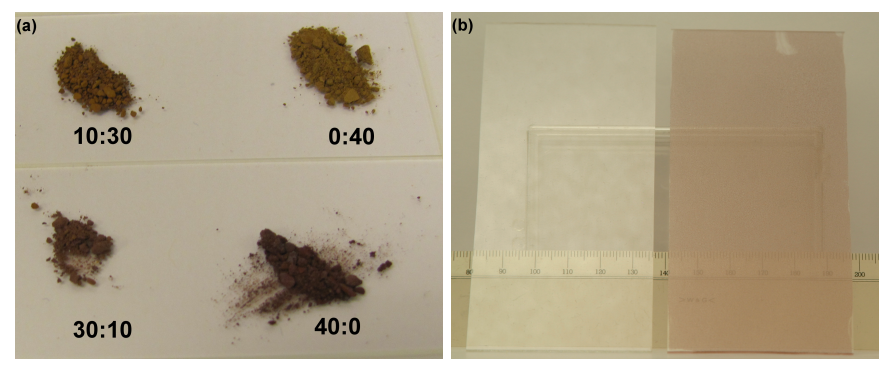

Figure 1: (a) Soil analogues used in this study. Notation indicates weight percentage of $\mathrm{Fe}_{2} \mathrm{O}_{3}$ :göthite. (b) Glass coupons with $0.20699 \mathrm{~g} / \mathrm{m}^{2}$ (left) and $2.82098 \mathrm{~g} / \mathrm{m}^{2}$ coatings of 40:0 grime. The scale behind the coupons is in $\mathrm{mm}$.

gion, relevant only to the bottom subcell in TJ devices. Yellow göthite was more wavelength dependent (FIG. 2a). In previous work (Burton and King, 2014b), we discussed the implications 
of wavelength dependent soiling on TJ devices. It was postulated that göthite rich soils may induce a current mismatch by reducing the available light in a narrow region, between 350 and $450 \mathrm{~nm}$. This peak overlaps a high energy portion of the AM1.5D spectrum (shaded in FIG. 2b). This is the onset for many devices, but is especially important to the top subcell. In addition to the current loss of the top subcell, the remaining subcells in series would be limited to a much lower current than nominally attainable if the cell current is limited by the top subcell.

Soil types common to desert regions with high PV market value often exhibit optical responses in the $300-900 \mathrm{~nm}$ range of the spectrum. Increasing the relative amount of yellow göthite

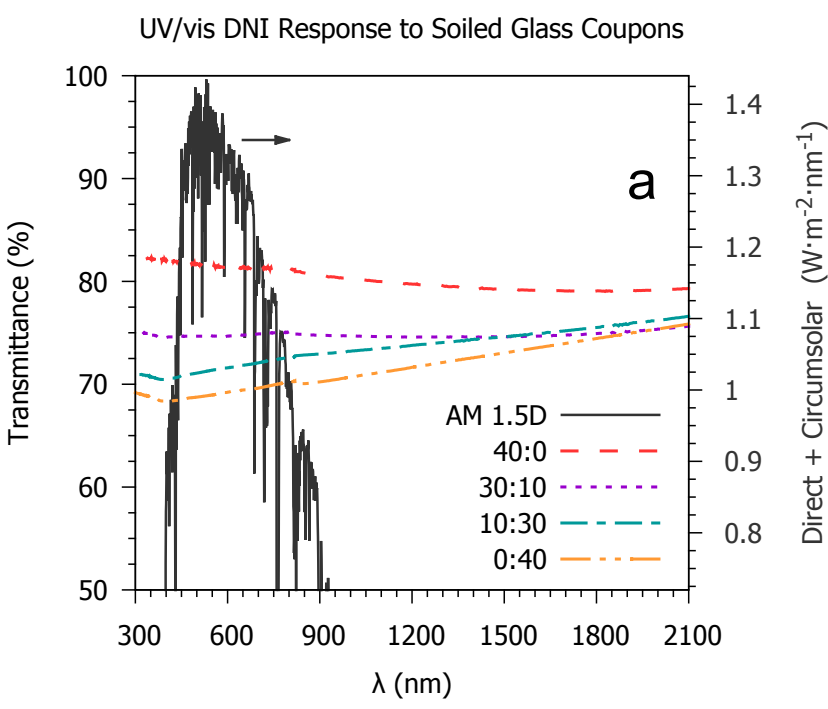

\section{UV/vis DNI Response to Glass Coupons with 0:40 Grime}

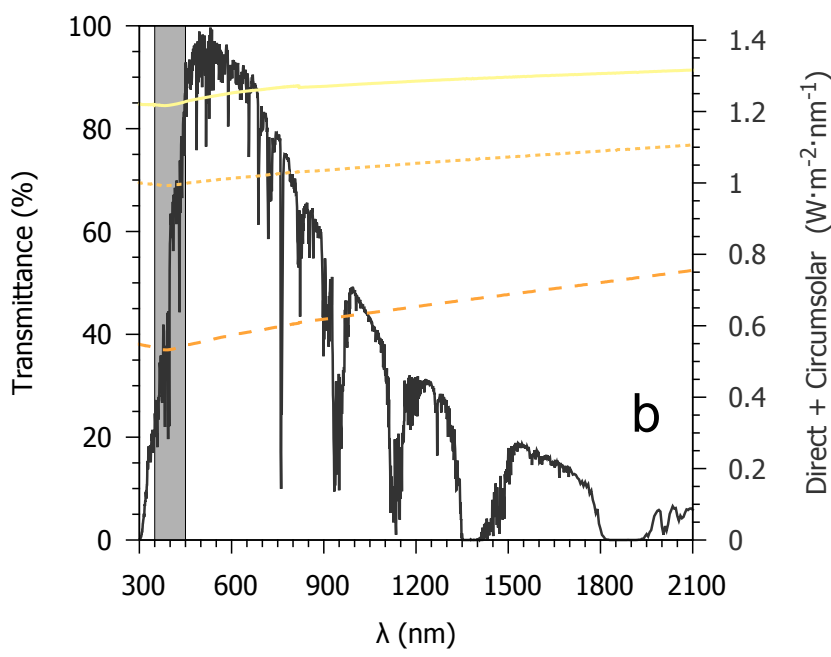

Figure 2: (a) UV/vis transmission through $\sim 1 \mathrm{~g} / \mathrm{m}^{2}$ grime films compared to AM1.5D (ASTM, 2012). (b) Typical response for 0:40 grime over range of tested samples $\left(0.22378,0.97063 \& 1.82657 \mathrm{~g} / \mathrm{m}^{2}\right.$ shown $)$

caused a decrease in direct optical transmission, especially at $425 \mathrm{~nm}$. The transmission through göthite-rich soils increased slightly at higher wavelengths (FIG. 2a). Göthite-rich soils ex- hibit characteristics which counter the most optimal operation of PV devices; they reduce transmission in the most energydense part of the spectrum, and are most transparent in the low energy region. The enhancement in collection over the lowenergy region is of little benefit, as the bottom subcell is never current limiting for GaInP/GaInAs/Ge type TJ cells. However; changes in the top or middle junction have a much more significant impact on the total power produced by the device, as illustrated by the schematic in FIG. 3.

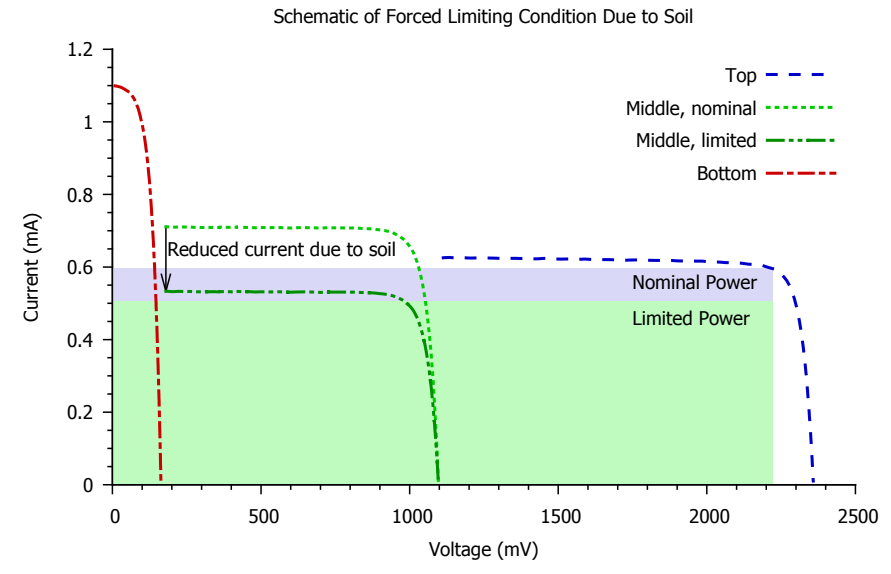

Figure 3: The decrease in current due to soil obscuring incident light may change the current balance of TJ devices. The resulting forced limiting condition would cause a decrease in power.

The percent transmission recorded by UV/vis spectroscopy, $\% T(\lambda)$, was used to predict decreases in $J_{\mathrm{SC}}$ due to soil accumulation on concentrator optics. The $\% T(\lambda)$ data was normalized to a clean glass coupon during data collection, and any other spectral effects were assumed to be constant between the reference condition and each test grime. The current density was calculated by integrating the predicted response of each subcell over the relevant wavelengths (Eq. (1)). As was inferred from FIG. 2a, the 40:0 red soil caused the lowest predicted loss in the top subcell of the isotype sensor, while increasing göthite content corresponded to variations in the current balance at AM1.5D. The bottom subcell showed a similar response to all soil types, indicating that the spectral behavior was less significant for this cell type.

The difference between the middle and top subcells was calculated and plotted as an excess current in FIG. 4. Any points above the dashed line signify a top subcell limiting condition, while points below the line indicate a middle subcell limiting condition. The 40:0 grime was predicted to show a very uniform behavior, exhibiting current matched behavior near AM1.5D, similar to the unsoiled reference. In contrast, the more spectrally responsive grime types were predicted to show much less uniform behavior. Most notably, the switch between top-limiting and middle-limiting behavior occurred over a wider range of airmass values for increasing göthite content. Additionally, the excess current did not follow mass loading exactly, but was instead a general trend (note the non-monotonic 
order of the curves for the 0:40 data set). The shift in limiting current behavior occurs within the prime energy collection window. For a real device with the same specifications as the isotype cells, this could lead to an extended period of top subcell limiting behavior.

This effect is most clearly illustrated at the summer and winter solstices. The changing seasons introduce deviations in the spectrum due largely to changes in airmass as the installation site rotates relative to the sun. This geometric variation has been the focus of other work describing solar elevation (McDonald and Barnes, 2008) and time of day (Torrey et al., 2011). Variation in spectrum can be compounded by the composition of accumulated soils. A series of DNI spectra was calculated in SMARTS using data gathered in Albuquerque, NM for two recent solstices, 06/20/2013 and 12/21/2012. The soil transmission data was applied to each spectrum as before, and the limiting current has been plotted for 40:0 and 0:40 in FIG. 5. The reference (unsoiled) data is shown in FIG 5a for illustration. The limiting current has been highlighted in green as the minimum of either the top (solid blue line) or middle (dashed red line) subcells. This convention will be used for the other plots in this figure, where only the limiting current will be shown for clarity. As was predicted from FIG. 4, the 40:0 grime showed a very consistent cross-over from top-limiting to middle-limiting between 8:00-8:30 and 15:30-16:00 on the summer solstice. A trend towards an increasing period of limiting conditions for increasing mass loadings (note the widening gap between curves, FIG. 5e) was noted. The 40:0 grime is nearly a neutral density filter, but is slightly more transparent to blue light (FIG 2a). As the mass loading increases, the top subcell collects the extra blue light, forcing the middle subcell into a current limiting condition earlier in the day.

Grime with a higher göthite content is less transparent to blue light, thus preventing the top subcell from operating at full capacity. In some cases, the 0:40 grime was predicted to prevent the top subcell from exceeding the current of the middle subcell throughout the entire day. However; this trend was not consistent with respect to mass loading, as the onset and duration of the limiting condition varied throughout the range (FIG. 5b). Mixtures of the soil types resulted in intermediate cell behavior. The middle subcell limiting condition was shifted to shorter periods later in the day as the mass loading of 10:30 grime (FIG. 5c) was increased. The proportionally greater göthite content restricted the transmission of blue light, but in a more consistent trend than the 0:40 samples. The 30:10 grime (FIG. 5d) was a near perfect balance, as all but the heaviest samples were predicted to undergo limiting behavior at the same time of day.

For the winter solstice, variations in spectrum due to soils were not predicted to cause any change in limiting current. Since the availability of blue light is lower in winter, the top subcell is more prone to limiting behavior than the middle subcell. Calculations with each soil type predicted that the top subcell would remain current limiting at all tested soil levels.

The difference between the soil types illustrates the significance of the incident spectrum on device performance. The red 40:0 grime did not induce as much spectral variation as the other test samples. As we discussed in prior work (Burton and
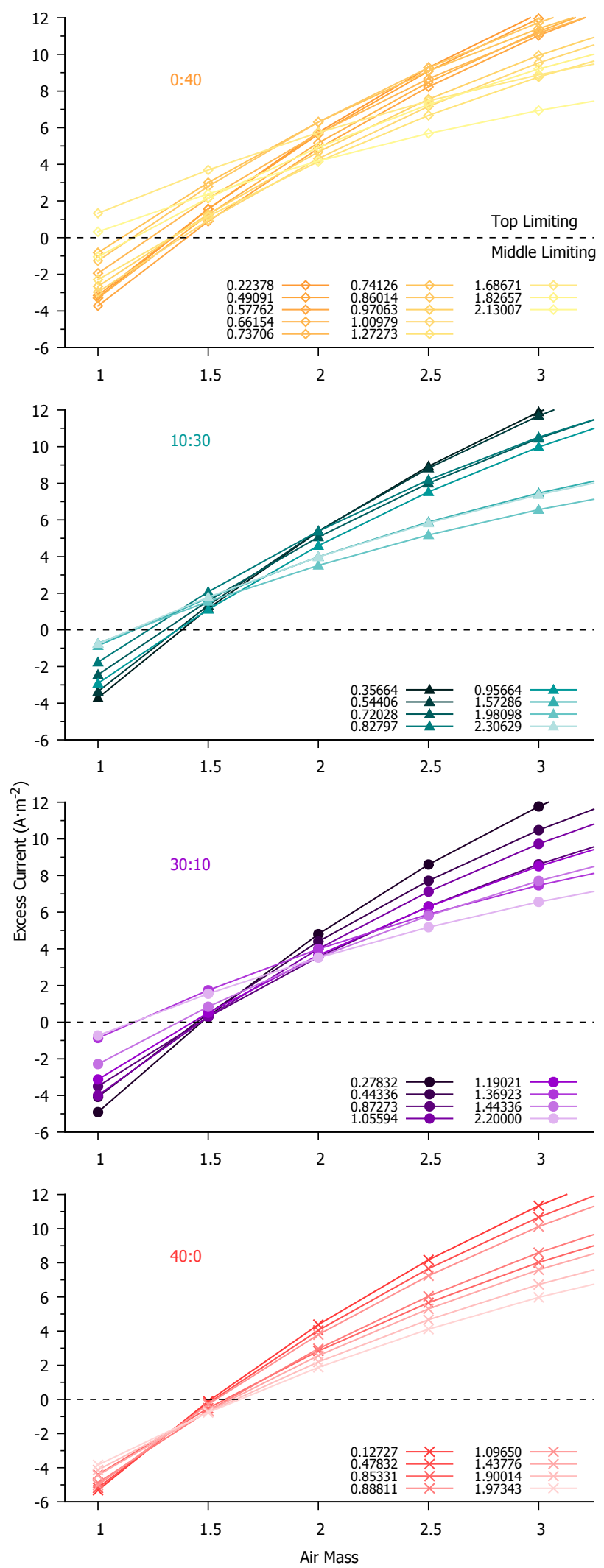

Figure 4: The excess current near AM2.0 is shown as a series of each grime type, calculated using standard SMARTS inputs and airmass values from 1 to 15. The mass loading of each sample is shown in $\mathrm{g} / \mathrm{m}^{2}$ in each key. The 40:0 grime shows very uniform behavior, while other samples show a much more erratic response. 

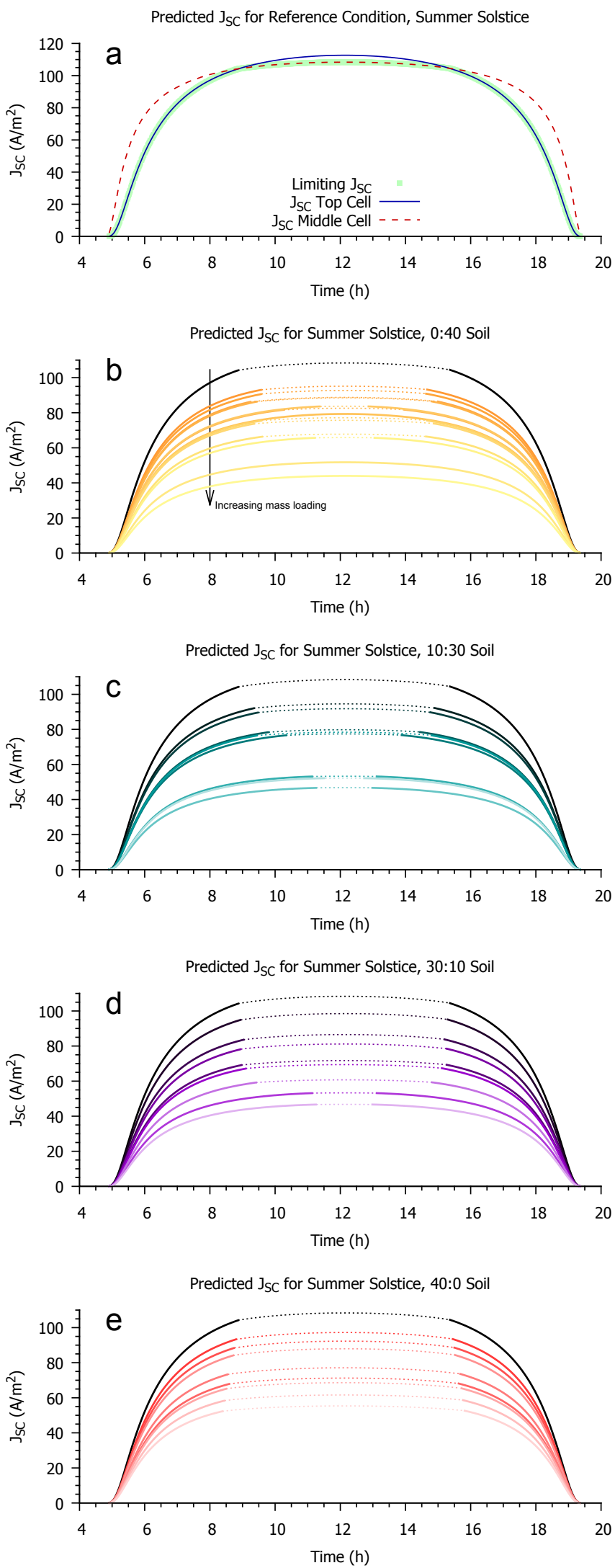

Figure 5: (a) The minimum of the top and middle sub-cells is shown highlighted as the limiting current. (b-e) The limiting current for the summer solstice is shown as a solid line for the top cell and a dashed line for the middle cell. Mass loading increases in order from black (clean reference) to lightly shaded for each data set.
King, 2014b), the relatively flat profile of the red soil made it an effective near-neutral density filter. The visually observed red color is due to reflection, which is not included in the DNI measurement. As a result, the spectrum passing through such a soil coating would reach the device relatively unchanged. Very heavy mass loadings would cause a slight change in cell behavior; however, this effect would be dwarfed by the magnitude of the total current loss due to reduced irradiance reaching the cell. In contrast, the yellow grime blends were much more prone to scattering light (Burton and King, 2014b), making the resulting measurements far less consistent and prediction more difficult. Fielded systems would likewise experience a much wider variation in incident spectrum than predicted by airmass alone. Unexpected changes in the resulting current would subsequently alter the maximum power point, further complicating optimal operation. A detailed analysis of the spectral effect on device performance could be significant to long-term installation planning.

\section{Conclusions}

We have demonstrated a method to model the spectral sensitivity of TJ cells to various types of grime corresponding in color to naturally occurring soils. Spectrally responsive yellow test grime had previously shown significant transmission decreases in the 300-600 nm range (Burton and King, 2014a). This region is of particular interest due to the natural spectral content of sunlight. Due to the spectral balance requirements of HCPV TJ cells, small fluctuations in spectral content could have an impact in the energy produced. Grime blends containing spectrally-responsive göthite were predicted to cause a much more varied response than the nearly-neutral density 40:0 grime. This behavior is most relevant to current balancing between the top and middle subcells.

The wide variation noted for spectrally responsive soil presents an additional challenge to performance modeling and monitoring. Long-term predictions may require a thorough site evaluation and soil analysis to obtain sufficient data. Site planning and allocation of cleaning resources may benefit from a more in-depth consideration of soil type and spectral response.

\section{Acknowledgments}

This work was supported by the U.S. Department of Energy SunShot Initiative. Sandia National Laboratories is a multiprogram laboratory managed and operated by Sandia Corporation, a wholly owned subsidiary of Lockheed Martin Corporation, for the U.S. Department of Energy's National Nuclear Security Administration under contract DE-AC04-94AL85000.

ASTM, 2012. Standard G173-03 Standard Tables for Reference Solar Spectral Irradiances: Direct Normal and Hemispherical on $37^{\circ}$ Tilted Surface. ASTM International, West Conshohocken, PA.

URL www . astm. org

Baig, H., Heasman, K. C., Mallick, T. K., 2012. Non-uniform illumination in concentrating solar cells. Renewable and Sustainable Energy Reviews $16(8), 5890-5909$. 
Burton, P. D., King, B. H., 2014a. Application and Characterization of an Artificial Grime for Photovoltaic Soiling Studies. Photovoltaics, IEEE Journal of 4 (1), 299-303.

Burton, P. D., King, B. H., 2014b. Artificial Soiling of Simulated Photovoltaic Module Surfaces using a Variety of Synthesized Soil Types. Photovoltaics, IEEE Journal of 4 (-), In Press.

Cotal, H., Sherif, R., 2005. The effects of chromatic aberration on the performance of $\mathrm{GaInP} / \mathrm{GaAs} / \mathrm{Ge}$ concentrator solar cells from Fresnel optics. In: Photovoltaic Specialists Conference, Conference Record of the Thirty-first IEEE. pp. 747-750.

El-Shobokshy, M. S., Mujahid, A., Zakzouk, A. K. M., 1985. Effects of dust on the performance of concentrator photovoltaic cells. Solid-State and Electron Devices, IEE Proceedings I 132 (1), 5-8.

Gueymard, C. A., December 1995 1995. SMARTS2, A Simple Model of the Atmospheric Radiative Transfer of Sunshine. Tech. Rep. FSEC-PF-270-95, Florida Solar Energy Center.

Gueymard, C. A., 2001. Parameterized transmittance model for direct beam and circumsolar spectral irradiance. Solar Energy 71 (5), 325-346.

Gueymard, C. A., 2009. Daily spectral effects on concentrating PV solar cells as affected by realistic aerosol optical depth and other atmospheric conditions. In: Optical Modeling and Measurements for Solar Energy Systems III. Vol. 7410. pp. 741007-741014.

King, R. R., Bhusari, D., Boca, A., Larrabee, D., Liu, X. Q., Hong, W., Fetzer, C. M., Law, D. C., Karam, N. H., 2011. Band gap-voltage offset and energy production in next-generation multijunction solar cells. Progress in Photovoltaics: Research and Applications 19 (7), 797-812.

McDonald, M., Barnes, C., 2008. Spectral optimization of CPV for integrated energy output. In: Proc. SPIE. Vol. 7046. pp. 704604-704612.

Miller, D. C., Kurtz, S. R., 2011. Durability of Fresnel lenses: A review specific to the concentrating photovoltaic application. Solar Energy Materials and Solar Cells 95 (8), 2037-2068.

Mingguo, L., Kinsey, G. S., Bagienski, W., Nayak, A., Garboushian, V., 2013. Indoor and Outdoor Comparison of CPV III-V Multijunction Solar Cells. Photovoltaics, IEEE Journal of 3 (2), 888-892.

Muller, M., 2009. Development of an Outdoor Concentrating Photovoltaic Module Testbed, Module Handling and Testing Procedures, and Initial Energy Production Results. Tech. Rep. NREL/TP-520-46569.

Qasem, H., Betts, T. R., Müllejans, H., AlBusairi, H., Gottschalg, R., 2012. Dust-induced shading on photovoltaic modules. Progress in Photovoltaics: Research and Applications, -

Stafford, B., Davis, M., Chambers, J., Martinez, M., Sanchez, D., 2009. Tracker accuracy: field experience, analysis, and correlation with meteorological conditions. In: Photovoltaic Specialists Conference, Conference Record of the Thirty-fourth IEEE. pp. 002256-002259.

Torrey, E. R., Ruden, P. P., Cohen, P. I., 2011. Performance of a split-spectrum photovoltaic device operating under time-varying spectral conditions. Journal of Applied Physics 109 (7), -.

Viana, T. S., Rüther, R., Martins, F. R., Pereira, E. B., 2011. Assessing the potential of concentrating solar photovoltaic generation in Brazil with satellitederived direct normal irradiation. Solar Energy 85 (3), 486-495.

Victoria, M., Herrero, R., Domínguez, C., Antón, I., Askins, S., Sala, G., 2013. Characterization of the spatial distribution of irradiance and spectrum in concentrating photovoltaic systems and their effect on multi-junction solar cells. Progress in Photovoltaics: Research and Applications 21 (3), 308-318. Vivar, M., Herrero, R., Antón, I., Martínez-Moreno, F., Moretón, R., Sala, G., Blakers, A. W., Smeltink, J., 2010. Effect of soiling in CPV systems. Solar Energy 84 (7), 1327-1335. 
Graphical Abstract014. This manusc99qut version is made available under the Elsevier user license

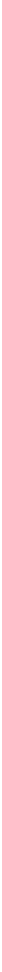

